\title{
Diacronie
}

Studi di Storia Contemporanea

$N^{\circ} 12,4 \mid 2012$

Sulle tracce delle idee

\section{Note sulla politica culturale del fascismo in Spagna (1922-1945)}

\section{Rubén Domínguez Méndez}

\section{Q OpenEdition}

1 Journals

\section{Edizione digitale}

URL: https://journals.openedition.org/diacronie/2503

DOI: $10.4000 /$ diacronie. 2503

ISSN: 2038-0925

\section{Editore}

Association culturelle Diacronie

\section{Notizia bibliografica digitale}

Rubén Domínguez Méndez, «Note sulla politica culturale del fascismo in Spagna (1922-1945)», Diacronie [Online], $N^{\circ} 12,4$ | 2012, documento 5, online dal 29 décembre 2012, consultato il 15 février 2023. URL: http://journals.openedition.org/diacronie/2503; DOI: https://doi.org/10.4000/diacronie. 2503

\section{(c)}

Creative Commons - Attribuzione - Non commerciale - Non opere derivate 4.0 Internazionale - CC BYNC-ND 4.0

https://creativecommons.org/licenses/by-nc-nd/4.0/ 


\title{
Diacronie
}

\section{Note sulla politica culturale del fascismo in Spagna (1922-1945)}

\author{
Rubén DOMÍNGUEZ MÉNDEZ*
}

Questo saggio analizza la politica culturale sviluppata dal fascismo italiano in Spagna nel periodo compreso tra la presa del potere in Italia fino alla fine della Seconda Guerra Mondiale. Attraverso questa politica si sviluppò la strategia di espansione e proselitismo del regime fascista nei confronti della società spagnola. Emergerà così l'interesse del fascismo per porre la cultura italiana allo stesso livello di quelle delle grandi Potenze. Questo progetto fu però condizionato dallo sviluppo politico interno alla Spagna. Nel testo vengono analizzati i diversi veicoli usati dal fascismo: le scuole italiane all'estero, gli Istituti Italiani di Cultura e i programmi di scambio e di cooperazione culturale.

\section{L'esportazione della cultura fascista}

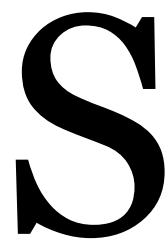

e prendiamo in considerazione l'intera epoca contemporanea ci rendiamo conto che l'interesse per il retaggio culturale è andato progressivamente aumentando, fino a diventare un meccanismo indispensabile per ottenere un riconoscimento internazionale. Nel caso del fascismo si adottarono misure culturali ed educative con l'obbiettivo di utilizzare le istituzioni statali oltrefrontiera come mezzi di propaganda. È da notare, tuttavia, che l'azione di propaganda culturale all'estero non fu direttamente incoraggiata dal governo italiano. Fu l'iniziativa privata, promossa da membri della comunità emigrata o da società nazionali presenti all'estero - come la Società Nazionale Dante Alighieri -, che assunse 
l'incarico di rappresentare i valori dell'Italia a partire dai decenni immediatamente successivi alla nascita dello Stato unitario.

Il fascismo non ebbe dubbi sull'opportunità di utilizzare la cultura come una vetrina ideale per il regime. L'azione presentava elementi di continuità, ma anche di innovazione rispetto al periodo precedente. Si può parlare di continuità a proposito della politica di istruzione sviluppata all'estero: la struttura scolastica, "eredità" degli anni precedenti, continuava ad operare nell'ambito del Ministero degli Affari Esteri, che l'aveva creata; le novità erano invece rappresentate da:

- l'aumento dei centri di istruzione elementare e media all'estero, sempre maggiore, sia sul piano geografico, sia riguardo al numero degli studenti e delle attività;

- la costruzione de una rete di IIC (Istituti Italiani di Cultura);

- l'avvio di una Direzione Generale per la Propaganda, all’interno del Minculpop, per svolgere le sue funzioni all'estero;

- la creazione dell'Istituto Nazionale per le Relazioni Culturali con l'Estero (IRCE) all'ínterno del Ministero degli Affari Esteri;

- la riproduzione delle istituzioni create in Italia anche presso comunità emigrate in altri paesi, come l'Opera Nazionale Dopolavoro (OND), l'Opera Nazionale Balilla (ONB) o i fasci ;

- la capacità di adattare questa azione culturale alle innovazioni tecnologiche del periodo con i nuovi mezzi di comunicazione di massa.

Inoltre, per ottenere il consenso della comunità italiana all'estero il regime fascista si valse principalmente di tre meccanismi: il fascio all'estero, il consolato e la Casa d'Italia (insieme al dopolavoro). La collaborazione tra questi organismi avrebbe messo in marcia le attività propagandistiche, culturali e ricreative.

\section{La ricezione del fascismo durante la dittatura primo rriverista}

L'influenza del fascismo italiano fu fondamentale nella formazione autoritaria della dittatura di Primo de Rivera. Allo stesso modo, le relazioni tra i due dittatori si intensificarono nell'intento di modificare la politica estera condotta dalla Francia finalizzato a creare un blocco fra i due paesi in chiave anti-francese. 
Sul piano culturale il fascismo non riuscì a creare nel paese strutture sufficienti per incrementare le attività culturali che aveva sviluppato lo Stato liberale italiano. Nella fase iniziale del fascismo italiano, la Spagna non può essere infatti considerata una priorità immediata tra le sue mire espansionistiche. Le scuole continuarono a finanziarsi attraverso le donazioni ed un sussidio dal Ministero degli Affari Esteri; non si fondarono in quegli anni nuove sedi dell' Istituto Italiano di Cultura (IIC), benché il fascismo avesse intrapreso un progetto espansivo a partire dal $1926^{1}$. La situazione portò allo sviluppo di un'azione culturale altamente informale e sfilacciata, che veniva affidata alla volontà dei singoli elementi: insegnanti, membri dei fasci o consoli. Una politica di interventi non disegnati che aveva nella città di Barcellona il centro d'azione. Due furono i tratti che caratterizzarono questa azione:

- La volontà di contrastare il peso culturale esercitato da altre nazioni europee, in particolare dalla Francia e dalla Germania;

- L'intenzione di stabilire le prime basi da cui sviluppare ulteriormente la politica culturale attraverso la diffusione di un messaggio che avrebbe dovuto combinare i valori della tradizione latina e della modernità fascista.

Il peso minore della cultura italiana nella vita intellettuale del paese risultava evidente tanto nel mondo accademico e scientifico, quanto negli altri ambiti. Così, in questa fase, furono pochi gli intellettuali chiamati nella Penisola iberica per impartire lezioni; si ridusse il numero di rappresentazioni di opere da parte di gruppi teatrali italiani; era esigua la diffusione di libri e periodici nel paese. Una situazione scoraggiante e che a volte sembrava, per assurdo, causata dagli stessi responsabili della promozione della cultura italiana:

È da consigliarsi che le pubblicazioni di propaganda e reclame siano redatte possibilmente in spagnolo od almeno in italiano, mai in francese, come quasi sempre accade anche per parti di entità ufficiali² ${ }^{2}$

\footnotetext{
${ }^{1}$ Questa situazione rimase praticamente invariata nel corso del periodo della dittatura di Primo de Rivera. Così, mentre le scuole laiche si concentravano esclusivamente a Barcellona, il governo di Mussolini estese la sua influenza culturale sovvenzionando centri religiosi in nuove aree geografiche in cambio della promozione della lingua italiana nelle loro aule.

2 Archivio Storico del Ministero degli Affari Esteri (ASMAE), Fondo Archivio Scuole (As.), 19231928, b. 656, Console Generale a Barcellona al direttore della Direzione Generale delle Scuole Italiane all'Estero (DGSE), Mazzini-Trabalza, 1 aprile 1925.
} 
Nel 1924 fu inviato a Madrid il professor Ruggero Palmieri - un uomo di lettere che conosceva la mentalità spagnola ed aveva scritto numerosi libri dedicati alla sua letteratura - con l'intento di schiudere strade attraverso le quali migliorare la diffusione della cultura italiana ed occuparsi della cattedra di italiano presso l'Università di Madrid. Palmieri prese come esempio il modello francese e le sue istituzioni:«Molto potrà giovarmi l'esperienza dell'Istituto francese che da parecchi anni vive di una vita prospera e efficace»3, ma la sua azione non fu sostenuta delle risorse economiche necessarie. Per questo motivo, il principale reddito per l'Italia arrivò con l'introduzione dell'insegnamento della lingua italiana all'interno del sistema scolastico spagnolo, in particolare nella scuola media, con il piano di studi promosso dal ministro Eduardo Callejo nel 1926.

Per quanto riguarda la comunità italiana nel paese, dobbiamo considerare che la colonia di Barcellona rappresentava in buona sostanza il 50\% della presenza nel paese, su un totale di circa 5.000 italiani censiti. A differenza di altre piccole comunità4, quella del capoluogo catalano aveva sviluppato, dopo la fine del XIX secolo, un certo numero di organismi in grado di proteggere i loro interessi attraverso la creazione di associazioni: Camera di Commercio, Società Reduci e Smobilitati, Società di Mutuo Soccorso, Società di Beneficenza, Opera Bonomelli e il Comitato della Società Dante Alighieri5. Il fascismo ebbe la necessità di introdurre in questi organismi la gerarchia fascista, ma doveva farlo con cautela, per evitare di generare contrasti con i dirigenti precedentemente in carica e senza provocare gravi rotture. Gli spazi che la tradizione associativa della comunità italiana aveva creato nella precedente fase liberale furono riorganizzati dal fascismo: si utilizzò l'esistente Casa degli Italiani per creare un patronato con il fine di raccogliere tutte le attività associative consentite e incoraggiate dal regime. Un'azione dotata di un grande significato simbolico che avrebbe dovuto definire l’immagine con cui si sarebbe dovuta identificare la comunità italiana. Si sperava di ottenere la riunificazione di tutte le attività coloniali: consolato, Camera di Commercio, scuole e associazioni. Il processo determinò la fusione degli organismi, anche se solo dopo aver superato le reticenze del capo del comitato della Dante, Benedetto Colarossi. Quest'ultimo vedeva nella misura, accettata dalla presidenza

\footnotetext{
3 ASMAE, As., 1923-1928, b. 671, Ruggero Palmieri al direttore de la DGSE (Trabalza), 15 aprile 1924.

4 CRESCIANI, Gianfranco, Fascismo, antifascismo e gli italiani in Australia (1922-1945), Roma, Bonacci, 1979, p. 13.

5 La Società di Beneficenza gestiva una scuola elementare mentre che il comitato della Dante aveva creato una scuola media orientata agli studi commerciali.
} 
centrale a Roma ${ }^{6}$, una diminuzione dell'importanza dell'ente che, nel frattempo oltre alle lezioni di italiano per spagnoli aveva iniziato i corsi di scuola media. La vicenda si concluse nel 1927, con l'avvicendamento di Colarossi con il console Mazzini: in questo modo si sarebbe cancellata la forte influenza che ancora esercitavano nella direzione della Casa i dirigenti prefascisti ${ }^{\text {. }}$

In seguito alla fusione delle scuole fu necessario stabilire un adeguato programma educativo per raggiungere gli obiettivi perseguiti dal fascismo. Coerentemente con questa linea le scuole italiane all'estero avrebbero dovuto rappresentare uno dei vettori principali per il trasferimento della nuova ideologia alle comunità emigrate. Per questo compito, sarebbe risultata fondamentale la collaborazione di coloro che erano incaricati di guidare il processo di apprendimento, ovvero gli insegnanti; quest'ultimi potevano concorrere, oltre che all'acquisizione di una conoscenza oggettiva da parte degli studenti, a inondare di propaganda le scuole con i programmi e le risorse didattiche; tutto ciò doveva essere poi documentato con un rapporto iniziale e un resoconto finale.

Quanto ai giovani, il 'simbolo' preferito del fascismo, si procedette al loro indottrinamento fascista attraverso un processo di nazionalizzazione e di organizzazione della scuola grazie al gruppo giovanile italiano di Barcellona, costruito sul modello della ONB. Prima ancora della sua nascita erano state già diffuse le disposizioni relative al corpo nazionale per l'educazione fisica per le scuole medie all'estero e presentate come un modo con cui promuovere lo sport e la ginnastica tra i giovani. A Barcellona, nel gennaio 1927, l'educazione fisica fu inclusa tra le materie che gli insegnanti italiani dovevano sviluppare durante l'anno scolastico: il doposcuola, la versione giovanile del dopolavoro popolare, mosse i primi passi con Orfeo Gallo come capo del gruppo.

\footnotetext{
${ }^{6}$ Archivio Storico della Società Dante Alighieri (ASSDA), Comitati Esteri (Ce.), Barcelona, f. 48, s. f. A, Riservata, Presidente del comitato della Dante a Barcelona al Segretario Generale della Dante, Colarossi-Zacagnini, 25 marzo 1923. Su questi argomenti si veda SALVETTI, Patrizia, Immagine nazionale ed emigrazione nella Società Dante Alighieri, Roma, Bonacci Editore, 1995; PISA, Beatrice, Nazione e política nella Società Dante Alighieri, Roma, Bonacci Editore, 1995.

7 Nel resto della comunità italiana nel paese, nelle altre città del Meditrraneo in cui erano visibili gli interessi economici delle élite italiana - Valencia, Maiorca, Cadice e si potrebbe includere a questo elenco anche Siviglia - insieme a la capitale Madrid, a Vigo e alle Isole Canarie, erano stati creati sezioni dei fasci italiani prima del 1927.
} 


\section{Il confronto ideologico con la Seconda Repubblica Spagnola (1931-1936)}

Le relazioni tra l'Italia e la Spagna mutarono significativamente dopo la caduta della monarchia. Si concluse in questo modo lo stretto rapporto che il governo spagnolo aveva mantenuto con l'Italia fascista. Con il consolidamento del potere nelle mani di Mussolini, il suo desiderio di stabilire una politica estera più ampia si tradusse nell'interesse a fornire copertura finanziaria e ideologica a gruppi disposti ad agire contro il nascente governo repubblicano. Nel campo della cultura, vennero aumentate le strutture educative, fu creato un IIC e l'uso dei lettori italiani presso le università spagnole come vettori di propaganda.

Gli spazi pubblici dell'emigrazione italiana subirono cambiamenti drastici a seguito dei cambiamenti politici che si verificarono in Spagna. Così, durante la Seconda Repubblica si constatò l'aumento della migrazione politica italiana con l'arrivo di un gran numero di fuorusciti italiani, in particolar modo a Barcellona. Questi risposero all'azione fascista ingaggiando una lotta per il controllo degli spazi di sociabilità e per impadronirsi della rappresentanza e l'essenza dell'italianità. Il risultato di questo confronto fu la riduzione dell'importanza dei fasci, che adottarono una strategia diversa da quella della diplomazia italiana.

Il fascismo, in parte anche ispirato all'esempio comunista, creò un organismo per sostenere l'espansione dell'ideologia nel mondo grazie alla cosiddetta internazionale delle camicie nere. Furono creati i Comitati d'Azione per l'Universalità di Roma (CAUR), con l'obiettivo di promuovere l'unione delle organizzazioni fasciste di altri paesi, dando orientamenti in materia di organizzazione o di propaganda da Roma, analogamente al ruolo svolto nel Comintern da Mosca. Inizialmente, le sezioni avrebbero potuto contare sul personale italiano dislocato nei rispettivi paesi che avrebbero fornito le indicazioni opportune. Tuttavia, ben presto si decise che i CAUR avrebbero dovuto essere composti esclusivamente da elementi autoctoni per evitare il conflitto con le autorità locali. In questo modo, nel 1934, nel momento in cui viene organizzato il Congresso Internazionale di Montreux, il gruppo che dava più sintomi di vicinanza al fascismo era Falange de las JONS. Quest'ultima, nel mese di ottobre, inviò un telegramma di congratulazioni al Duce per il tredicesimo anniversario della marcia su Roma, firmato da Ernesto Giménez Caballero e Jose Antonio Primo de Rivera in qualità di rappresentanti del CAUR; un mese dopo, il programma presentato dalla 
Falange fu definito da Celesia come neo-fascista, composto da dichiarazioni dottrinali «derivate o del Fascismo Italiano o dal Nazionalsocialismo tedesco»8.

Tra le novità della politica culturale italiana dell'epoca, si deve sottolineare, in primo luogo, lo sforzo realizzato per migliorare o modificare l'immagine che la società spagnola aveva del fascismo, soprattutto in considerazione del fatto che il governo repubblicano si basava su ideali che constrastavano con l'ideologia proveniente dall'Italia. Lo strumento chiave per raggiungere questo scopo fu quello di far circolare notizie sulla stampa spagnola finalizzate a pubblicizzare il movimento. Il secondo luogo si operò un tentativo volto a riconquistare la posizione che l'insegnamento dell'italiano aveva acquisito nei programmi della scuola media spagnola dal 1926. Infine, gran parte degli sforzi furono rivolti a creare centri per la diffusione della cultura italiana nel paese; una misura i cui risultati, per mancanza di realismo, non risultarono tanto ragguardevoli quanto lo sforzo investito in questa.

La sostituzione di Durini Di Monza non significò la caduta nell'oblio di queste linee guida; il suo sostituto, Raffaele Guariglia, fu uno degli ambasciatori italiani più attivi tra quelli che lavorarono a Madrid. Oltre a stabilire legami con i gruppi che cospiravano contro la Repubblica - probabilmente perché questa era la sua missione - avviò diverse iniziative per incrementare la presenza della cultura italiana nel paese. Nell'agosto del 1932 aveva ricevuto istruzioni da Mussolini per mettere in piedi una sorta di doppio gioco con i politici e le istituzioni repubblicane; a novembre cominciò a inviare informazioni sul potenziale della cultura per perseguire i fini perseguiti dal fascismo: questo avrebbe evitato di esporsi con riferimenti propagandistici diretti, che difficilmente sarebbero stati accettati in Spagna a causa dell'identificazione della dittatura primorriverista con lo Stato totalitario italiano. I modi con cui introdurre gradualmente gli spagnoli alla «comprensione del Fascismo come fatto politico di interesse universale o indispensabile elemento di cultura politica» erano ${ }^{9}$ :

- La partecipazione italiana alla vita universitaria spagnola attraverso la creazione di un istituto, così come aveva fatto la Francia attraverso la Casa de Velázquez;

- La promozione di un centro culturale o un'associazione italo-spagnola per la promozione di convegni e incontri tra intellettuali e studenti di entrambi i paesi;

- Lo sviluppo di accordi tra le università dei due paesi per la diffusione delle rispettive lingue.

8 ASMAE, Affari Politici (Ap.), 1931-1945, Spagna, b. 6, Adetto commerciale a Madrid al Ministro degli Affari Esteri, Celesia-Mussolini, 29 novembre 1934.

9 ASMAE, Ap., 1931-1945, Spagna, b. 3, Ambasciatore d'Italia a Madrid al Ministro degli Affari Esteri, Guariglia-Mussolini, 30 novembre 1932. 
Sulla prima, la creazione di un istituto, lo stesso Guariglia era consapevole del grande sforzo economico che doveva essere richiesto a Roma per l'acquisto dei terreni e le successive azioni per la costruzione dell'edificio: spese che lo Stato non era in grado di affrontare. Dovendo mascherare l'azione italiana sotto le spoglie culturali si progettò la creazione di un IIC in due possibili ubicazioni: Madrid -approfittando della recente creazione della Casa d'Italia nella capitale- o Barcelona. Fu scelta Barcellona, malgrado la situazione politica, in considerazione del fatto che il fascismo aveva lì più risorse $\mathrm{e}$ accoliti.

Il centro culturale si costituì come un organismo di propaganda utilizzato per promuovere un'immagine favorevole dell'Italia, sopratutto a seguito dell'invasione dell'Etiopia. Nella stessa prospettiva si deve interpretare l'intenzione di istituire nuovi comitati della Società Dante Alighieri: ad esempio quello stabilito a Bilbao grazie all'azione del console a San Sebastián; il diplomatico intendeva divenire il referente di questa società nella Spagna settentrionale. Per quanto riguarda l'insegnamento dell'italiano, il piano Callejo era stato sospeso a seguito del decreto del 13 maggio 1931, in cui tornava in uso il curriculum di studi del 1903. La nuova visione dell'istruzione era riflessa dal progetto di legge preparato da Lorenzo Luzuriaga il 17 novembre 1932. Per gli interessi italiani la questione era di grande importanza perché nella scuola media veniva escluso l'italiano tra le lingue moderne, mentre il francese era fissato come obbligatorio e l'inglese e il tedesco divenivano facoltativi ${ }^{10}$. Gli ambasciatori che si susseguirono tentarono di modificare questa situazione -senza successo- sondando la possibilità di stabilire un trattato culturale: questo vide la luce - peraltro soltanto come semplice progetto d'accordo - nel $1935^{11}$.

\section{Gli anni d'oro della politica culturale fascista durante il primo Franchismo (1936-1943)}

I militari golpisti cercarono sostegno all'estero e rivolsero una richiesta di aiuto ai governi stranieri che avevano fatto dell'autoritarismo il loro fondamento. Mussolini rispose in modo positivo alla petizione. Nella Guerra Civile tutta l'assistenza dell'Italia

${ }^{10}$ I contatti con il Ministerio de Asuntos Exteriores avevano fatto capire all'ambasciata italiana che ci sarebbero grosse difficoltà per modificare questa situazione. Archivo del Ministerio de Exteriores de Madrid (AMAE), Renovado, legajo 742, expediente 141.

${ }^{11}$ I continui cambi nel ministero misero in difficoltà alcune azioni. ASMAE, As., 1929-1935, b. 882, Direttore de la Direzione degli Italiani all'Estero (DIE) al Adetto commerciale a Madrid, Parini-Geisser Celesia, 14 maggio 1935. 
fascista fu coordinata attraverso l'Ufficio Spagna, creato all'interno del Ministero degli Affari Esteri sotto la direzione di Lucca Pietromarchi. Il contributo del fascismo al bando nacional - anche in termini di uomini e mezzi - vide nella propaganda una delle armi più potenti, poiché si valse di mezzi di comunicazione di massa come il cinema e la radio per tentare di vincere la battaglia dell'opinione pubblica e consolidare i risultati militari.

Nella capitale dei ribelli, Salamanca, la Missione Militare Italiana in Spagna (MMIS) organizzò un ufficio stampa e propaganda che agiva in modo indipendente della rappresentanza diplomatica, diretta da Roberto Cantalupo dal marzo 1937. Questo ufficio, in un primo momento coordinato dal giornalista Guglielmo Danzi ${ }^{12}$, era diviso in cinque sezioni: Sezione Spagna, Sezione Esteri, Sezione Radio e Legionario, Sezione Truppe Volontarie e Sezione Fotocine ${ }^{13}$. Dei tre mezzi di comunicazione utilizzati giornali, radio e cinema - fu particolarmente rilevante l'uso della stampa, che compendiava le notizie apparse nei vari quotidiani nel settimanale «Il Legionario» il 19 marzo $1937^{14}$. Una volta installata la MMIS sappiamo, da un documento redatto nel maggio 1937, della prospettiva dei rappresentanti italiani sul futuro della politica culturale da seguire in Spagna. Lo scopo era quello di contribuire alla penetrazione ideologica fascista grazie alle circostanze storiche che si sarebbero presentate in pochi mesi e sulla base dei «rapporti di cordialità che passano oggi tra l'Italia e la Spagna» ${ }^{15}$. Per ottenere ciò furono intraprese iniziative che avevano come obiettivo:

- ottenere il riconoscimento, da parte del governo di Burgos, della lingua italiana come elemento obbligatorio nell'istruzione nelle scuole secondarie;

- organizzazione di corsi di lingua italiana elementare nel maggior numero di città possibile;

12 «Danzi sería destituido en julio de 1937 y ocupará su cargo interinamente su segundo, Lamberti Sorrentino, hasta el nombramiento de Carlo Bossi que había sido cónsul general de Italia en Barcelona». PIZARROSO QUINTERO, Alejandro, «Intervención extranjera y propaganda: La propaganda exterior de las dos Españas», in Historia y Comunicación Social, 6/2001, p. 71.

13 ASMAE, Ap., 1931-1945, Spagna, b. 31, Ordinamento interno del Ufficio, 14 maggio 1937. Il documento contiene i nomi di tutto il personale assegnato a ciascun ufficio.

${ }_{14}$ Scritto in italiano, dapprima apparve con il titolo in spagnolo El Legionario, e con il sottotitolo di Giornale dei lavoratori combattenti in Spagna in difesa della civiltà europea, contro la barbarie rossa; dal 3 settembre 1937 si prese il nome Il Legionario, con il sottotitolo di Quotidiano dei volontari italiani combattenti in Spagna. Su questo particolare vid CORTI, Paola, PIZARROSO QUINTERO, Alejandro, Giornali contro: Il Legionario e II Garibaldino : La propaganda degli italiani nella guerra di Spagna, Alessandria, Edizioni dell'Orso, 1993.

15 ASMAE, Ap., 1931-1945, Spagna, b. 31. A nostro parere questo modifica lo dichiarato da Gianluca André sul fatto che non vi era nessun programma concreto per fascistizzare la Spagna. ANDRÉ, Gianluca, «L'intervento in Spagna e la politica estera fascista», in ESPADAS BURGOS, Manuel (coord.), Italia y la Guerra Civil Española, Madrid, CSIC, 1986, p. 25 
- istituzione di lettorati di lingua e letteratura italiana presso le università.;

- accordi per lo stabilimento di concessioni speciali con cui promuovere l'introduzione del libro italiano in Spagna, con l'idea di segnare nel futuro un accordo commerciale tra i due paesi.

Per intraprendere questo compito si considerò opportuno nominare gli insegnanti Paretti ed Ernesto Marchiandi come rappresentanti degli interessi italiani, rispettivamente, a Burgos - sede della Junta Técnica del Estado e residenza di Franco, dall'ottobre 1937 - e a Vitoria, nella cui Escuela de Artes y Oficios si istallò in modo provvisorio il Ministerio de Educación Nacional creato il 30 gennaio 1938. Una collaborazione in linea con il viaggio di Farinacci in Spagna per offrire consigli a Franco circa i percorsi da intraprendere nel futuro per la ricostruzione del paese ${ }^{16}$. Il ministro Pedro Sainz Rodríguez accettò la presenza di questi tecnici, fino al punto da tenere un incontro presso la sua stessa residenza il 18 settembre 1938, con la volontà di seguire personalmente la gestione delle suddette questioni ${ }^{17}$.

L'11 settembre 1937, durante la guerra civile, mascherando la partecipazione tedesca ed italiana nel Comitato internazionale di non-intervento, Mussolini annunciò la decisione di abbandonare la Società delle Nazioni. Adottata tale misura, nel mese di gennaio del 1938, l'ufficio mantenuto dall'Italia a Ginevra nel Comitato per la cooperazione intellettuale fu modificato per iniziativa di Ciano- in quel momento ministro degli affari esteri - con la creazione dell'IRCE (Istituto per le Relazioni Culturali con l'Estero). Il nuovo istituto fu proiettato alla promozione della cultura italiana con un approccio esageratamente nazionalista, che ambiva a promuovere il modello di organizzazione politica e sociale del fascismo. Per raggiungere questi obiettivi, l'IRCE favorì i contatti con organizzazioni straniere che avevano obiettivi simili, in particolare dopo lo scoppio della seconda guerra mondiale. Per le sue attività l'IRCE era in conflitto di competenze con il Ministero della Cultura Popolare (Minculpop), questione che non fu mai risolta. La Spagna, primo scenario dove potevano svolgere la loro azione, mostrò che i supposti problemi di sovrapposizione di competenze dell'IRCE era la mancanza di coordinamento che minacciava il predominio

${ }^{16}$ Secondo Coverdale, Farinacci mostrò a Franco l'opportunità di creare, sotto la sua autorità un Partido Nacional Español «que debería adoptar inmediatamente una actitud favorable hacia la clase obrera, garantizar la reforma agraria, la jornada de ocho horas, los sindicatos nacionales, el seguro de enfermedad, las pensiones de vejez, etc». COVERDALE, John, La intervención fascista en la Guerra Civil española, Alianza, Madrid, 1979, p. 181. Roberto Cantalupo dichiarò che non doveva interferire nella missione di Farinacci. CANTALUPO, Roberto, Fu la Spagna. Ambasciata presso Franco : Febbraio-Aprile 1937, Milano, Mondadori, 1948, p. 146.

${ }_{17}$ ASMAE, Direzione Generale delle Relazioni Culturali (Dgrc.) As., II versamento, 1925-1945, b.71, Ambasciata a San Sebastiano alla DIE, 25 agosto 1939. 
della cultura fascista nei rapporti con il bando nacional a vantaggio della diplomazia nazista ${ }^{18}$.

Analizzando i vari settori della cultura italiana nel paese, emerge come le scuole italiane soffrirono subito gli effetti della guerra. Con la partenza degli studenti e degli insegnanti per l'Italia, la DIE dovette avviare un processo complicato per convalidare i dati accademici di ogni studente e permettere loro di proseguire l'istruzione in Italia. D'altra parte, nelle zone della Spagna controllate dai ribelli si istituirono - con la collaborazione dei consoli, degli insegnanti e dei fasci - corsi di italiano elementare per l'anno accademico 1937/1938.

Nel dopoguerra la diplomazia culturale delle grandi potenze sembrava destinata a partecipare a una corsa per occupare posizioni privilegiate nella Spagna di Franco. Tuttavia, il risultato della guerra, consacrando la dittatura filofascista, creò una situazione favorevole a due Stati, Germania e Italia, che ne inondarono gli spazi pubblici di propaganda. La Germania, divenne così il rivale più duro per il fascismo per la convergenza ideologica che si sarebbe potuta stabilire con la Spagna19. Per "vincere" questa gara l'Italia sviluppò un vasto programma culturale, istituendo un IIC a Madrid - accompagnato dalle sezioni e delegazioni sparse nelle principali città - che fu la rete con cui radicarsi all'interno della società spagnola. Sei anni più tardi, la situazione italiana sarebbe stata molto diversa da quella riferita nella primavera del 1937 da Wilhelm Petersen - direttore del Centro de Intercambio Intelectual Germano-Español a Madrid - quando disse che l'Italia «carecía de unos presupuestos culturales básicos» per disporre di istituti e di scuole italiane nel paese $^{20}$. Furono messe in piedi otto sezioni - Madrid, Barcellona, Salamanca, San Sebastián, Siviglia, Valenza, Valladolid e Saragozza - con dodici delegazioni: Bilbao, Burgos, La Coruña, Gijón, Granada, Malaga, Oviedo, Pamplona, Santander, Santiago di Compostela, Tetuán e Vigo.

\section{Il declino dell'Italia fascista in Spagna (1943-1945)}

L’aggressività della politica estera della Germania e dell'Italia finirono per rompere il precario equilibrio internazionale stabilito dagli accordi di pace che avevano messo

\footnotetext{
18 ASMAE, Gabinetto, b. 1445, Direttore del IRCE al Direttore del Ufficio Spagna, De FeoPietromarchi, 28 maggio 1938.

19 Riguardo alla propaganda politica favorevole al regime nazista in Spagna (1936-1945), si veda: Les archives secrètes de la Wilhelmstrasse, vol 3, Paris, Plon, 1952.

${ }^{20} \mathrm{AL}$ contrario, indicava che l'Italia disponeva di «mayores presupuestos económicos para estas actividades». HERA MARTÍNEZ, Jesús de la, op. cit., p. 372. Non crediamo che Petersen ignorasse la politica educativa italiana nel paese prima della guerra civile o l'esistenza dell'IIC di Barcellona.
} 
fine alla Grande Guerra. L'incapacità della Società delle Nazioni nel risolvere i conflitti si inserì all'interno di un periodo caratterizzato dalla corsa agli armamenti e dalla crisi delle democrazie liberali. Con la vittoria di Franco e la riorganizzazione dello Stato ispirato agli esempi fascisti e nazista, la volontà di Mussolini di impegnarsi in una politica di potere nel paese lo portò a creare una rete di istituzioni culturali di vaste proporzioni. Pochi potevano presagire - solo quelli che vedevano nella decisione di schierarsi a fianco della Germania nazista l'inizio di un percorso di non ritorno- che questo lavoro sarebbe stato smantellato o ridotto a una dimensione minima solo cinque anni dopo e che il franchismo avrebbe abbandonato molti aspetti dell'estetica fascista nella sua strategia di consolidamento del potere.

Mussolini vide come il sistema che aveva creato finì per divorarlo il 25 luglio 1943. Quel giorno fu fissata la seduta del Gran Consiglio e nel corso della riunione i gerarchi mostrarono l'opposizione alla presenza di Mussolini alla direzione dello Stato. Al suo posto venne nominato come nuovo capo del governo il generale Pietro Badoglio: la guerra continuava sotto la guida suprema del $\mathrm{re}^{21}$. Durante questo periodo, sotto la pressione dei gruppi di opposizione al fascismo - che cominciavano a riorganizzare la loro attività - e delle richieste degli Alleati, Badoglio iniziò, tramite colloqui segreti, a stabilire un accordo di pace e Mussolini fu imprigionato per ordine del nuovo governo. La realtà italiana iniziò a chiarirsi solo dopo l'8 settembre, con l'annuncio dell'armistizio.

Nel campo delle relazioni culturali tra le due dittature i cambiamenti in ambito internazionale impedirono che fosse firmato l'accordo elaborato durante i primi mesi del 1943 e che aveva gettato le basi per rafforzare la cordialità di rapporti tra i due Stati. La notizia della creazione dei ministeri nella RSI fu diffusa a Madrid il 26 settembre. Per il Governo spagnolo la nascita della RSI veniva a creare una situazione complessa per via del mutato atteggiamento nei confronti degli Alleati ${ }^{22}$. Per evitare complicazioni internazionali la RSI non fu riconosciuta ufficialmente, anche se si inviarono istruzioni per il console a Milano, Fernando Canthal, affinché agisse con prudenza e si presentasse come il collegamento tra la RSI e la Spagna ${ }^{23}$. Sembrava lontano l'impegno di Franco con l'Asse, avviato con la ratifica dell'adesione all'AntiComintern il 27 marzo

${ }^{21}$ Gli eventi italiani furono seguiti in Spagna: «sin excesiva conmoción (...) Hasta el 27 de julio no se dio cuenta en España de la caída de Mussolini; se hizo incluso con fórmulas vagas y perífrasis que intentaban ocultar la gravedad de la crisis». ESPADAS BURGOS, Manuel, Franquismo y política exterior, Madrid, Rialp, 1987, p. 134.

${ }_{22}^{2}$ ALBONICO, Aldo, «La Spagna tra Badoglio e Mussolini (1943-45)», in Nuova Rivista Storica, 69, 3-4/1985, pp. 232-235.

${ }_{23}$ Canthal mostrò il suo apprezzamento per l'accoglienza che avevano le istruzioni impartite dal ministero sulla sua missione presso la Repubblica Sociale Italiana (RSI). ASMAE, RSI, b. 81, Console di Spagna a Milano al Ministero degli Affari Esteri, 30 novembre 1943. 
1939, confermato con l'adozione della non belligeranza nel giugno 1940 e la riunione tenutasi a Hendaya con Hitler il 23 ottobre di quello stesso anno. Era il momento di tornare alla posizione di partenza, cioè alla neutralità 24 . La ricomposizione del ministero degli Affari Esteri fu inoltre indice della volontà di Franco di mutare l'atteggiamento nei confronti degli Alleati, che già dominavano il Nord d'Africa. Il 3 settembre 1942 Serrano Súñer fu sostituito agli Esteri da Jordana: scompariva il ministro più incline all'alleanza con i totalitarismi nazista e fascista ${ }^{25}$. Secondo Ismael Saz si possono distinguere tre fasi nella relazione tra politica estera del franchismo ed evoluzione del ruolo della Falange. Inizialmente si riconosce una fase fascista tra il 1939 e il 1941: «en la que la guerra mundial y los éxitos del Eje favorecen una aceleración del proceso de fascistización»; questa perdura fino al 1942 quando, «todavía victoriosas las armas del Eje, la falange fascista sufre su definitiva derrota»; l'ultimo periodo vide la «aceleración del proceso de desfascistización» 26 .

Dal settembre 1943 le due Italie cercarono di ottenere l'ampia rappresentanza diplomatica in Spagna, alla quale si dovevano assommare gli insegnanti delle scuole e del'IIC; complessivamente il personale stipendiato dal Ministero degli Affari Esteri superava i 500 impiegati nel paese. La RSI non riuscì a convincere l'ambasciatore Paulucci - quando fu chiamato il 18 settembre 1943 - ad occupare il Ministero degli Affari Esteri della RSI. Questa circostanza fece sì che l'ambasciata e praticamente la totalità dei consolati optassero per restare vicino al governo Badoglio. Le difficoltà nel reperire una persona in grado di ricoprire questo ruolo in Spagna fecero sì che solo a novembre si potesse individuare nella figura di Eugenio Morreale il rappresentante della RSI. Per quanto riguarda il finanziamento delle attività, l'agenzia fascista creata fu

${ }^{24}$ La presunta capacità di Franco di tenere fuori della guerra alla Spagna - che, accanto alla teoria del tradizionale isolamento del paese dalla politica internazionale, fu molto pubblicizzata dalla storiografia del regime - è totalmente confutata. Mentre Hitler comprendeva come i benefici derivati della partecipazione spagnola sarebbero stati pochi - i possedimenti nel Marocco, a scapito del regime collaborazionista di Vichy - Mussolini non poté o non volle convincere Franco ad un'immediata entrata nella guerra dopo l'incontro a Bordighera il 12 febbraio 1941. Su quest'ultimo argomento vi sono valutazioni diverse. Cfr. quella di Javier Tusell sul possibile rivale nel Mediterraneo che il fascismo italiano avrebbe trovato nella Spagna per le compensazioni di guerra (TUSELL, Javier, Franco, España y la II Guerra Mundial. Entre el eje y la neutralidad, Madrid, Temas de Hoy, 1995) e quella, opposta, di Gennaro Carotenuto che, respingendo la paura della concorrenza nel Mediterraneo, considera dimostrata la mancanza di interesse dimostrato da Mussolini per la difficile situazione dell'esercito spagnolo (CAROTENUTO, Gennaro, «La carta spagnola. Mussolini e la Spagna durante la seconda guerra mondiale», in Spagna Contemporanea, 15, 1999, pp. 69-92). Il testo della riunione di Bordighera è disponibile nei Documenti Diplomatici Italiani (DDI), IX Serie, vol. VI, doc. 568 . ${ }^{25}$ Secondo Carotenuto, Suñer non fu così utile per il fascismo per il suo progressivo filonazismo. CAROTENUTO, Genaro, Franco e Mussolini, Milano, Sperling \& Kupper Editori, 2001, pp. 6568.

${ }^{26}$ SAZ CAMPOS, Ismael, Fascismo y Franquismo, Valencia, Universidad de Valencia, 2004, p. 161. 
sovvenzionata attraverso l'aiuto tedesco, mentre i britannici e gli americani fecero lo stesso con la rappresentanza monarchica. Nel dicembre 1943, Morreale si trasferì a Berlino per farsi dettare le linee da seguire nella riorganizzazione della rappresentanza della RSI in Spagna.

Mentre tutto ciò accadeva, l'epurazione dei rappresentanti italiani all'estero motivò il ritorno in Italia di quasi tutti i consoli e ambasciatori nominati durante il fascismo, anche se avevano successivamente dimostrato la loro fedeltà alla corona. Paulucci non rappresentò un'eccezione, malgrado quest'ultimo non condividesse questa decisione ${ }^{27}$. Il sostituito fu Tommaso Gallarati Scotti, scrittore e diplomatico, membro della nobiltà e cattolico modernista, firmatario del famoso "Manifesto degli Intellettuali Antifascisti”. Pur ottenendo la carica a Madrid, Gallarati Scotti non assunse l'incarico fino a metà febbraio del $1945^{28}$. Furono dettate istruzioni per collaborare con la Spagna in quanto nazione latina e nel contesto dei rapporti con gli Alleati, sia al nuovo incaricato d'affari, Luciano Mascia, sia all'ambasciatore. Gallarati Scotti doveva tentare di risolvere due problemi: il prestito italiano erogato durante la guerra civile e le navi bloccate nei porti spagnoli. Nel campo delle istituzioni culturali abbiamo sottolineato come l'IIC seguì gli orientamenti che erano stati stabiliti da Paulucci. In tal modo, si prese atto di non poter sostenere i costi delle numerose sedi. La prima riduzione si produsse nel corso del biennio 1943-1944, quando passò da 65 professori a 44 con l'eliminazione di 8 delegazioni: Bilbao, Burgos, La Coruña, Gijón, Malaga, Pamplona, Santander e Tetuán; restarono aperte tutte le sedi nelle città universitarie -dove poteva continuare l'esperienza dei lettorati - oltra a San Sebastián e Vigo. Con il cambiamento di linea le spese furono ridotte di oltre un milione rispetto all'anno precedente, ma riprendendo le parole dell'ambasciatore, se non era stata scelta una soluzione più radicale era semplicemente per non «interrompere gli studi a centinaia di allievi con conseguente diminuzione di prestigio per il Regio Governo» ${ }^{29}$.

Così, Paulucci considerava opportuno mantenere attive le scuole italiane a Madrid e Barcellona, ma credeva fosse necessario trasformare l'IIC in un istituto ispano-italiano

27 ASMAE, Personale, Serie I, Diplomatici e consoli, b. 29, f. 2/Paulucci di Calboli, Segreto, Ambasciatore dell'Italia a Madrid al Sottosegretario degli Affari Esteri, Paulucci-Visconti Venosta, 15 agosto 1944.

${ }^{28}$ Secondo le informazioni fornite dalla RSI, il governo spagnolo si sarebbe opposto in un primo momento alla nomina osservando come Gallarati Scotti non fosse un ambasciatore di carriera, ma un rifugiato politico all'estero; solo dopo forti pressioni dal Vaticano venne accettata la sua nomina in Spagna.ASMAE, RSI, b. 38, Appunto per il Duce, 23 settembre 1943.

29 Per 1942/43 erano stati spese 2.850.000 pesetas, mentre per 1943-1944 - secondo i dati inviati nel mese di febbraio - si prevedeva di spendere 1.597.260 pesetas mensili così ripartite: IIC 60.490 pesetas, scuole di Madrid 47.135 pesetas e scuole di Barcellona 25.480 pesetas. ASMAE, Dgrc. As., I versamento, 1936-1945, b. 116, Ambasciatore a Madrid al Ministro degli Affari Esteri en Brindisi, Paulucci-Badoglio, 25 febbraio 1944. 
che «potrebbe togliere all'istituto quel carattere di propaganda unilaterale [...] ed avrebbe il vantaggio di assorbire autorità e personalità del mondo culturale locale»30. Infine, tra le riforme progettate da Paulucci, emergeva l'intenzione di abolire i lettorati presso tutte le università, tranne quella di Madrid che sarebbe ricaduta sotto la tutela del direttore italiano del progettato istituto italo-ispanico. Come risultato, dai 44 insegnanti ancora attivi si passò a 14, così suddivisi: la scuola di Madrid avrebbe richiesto 5 professori e 3 maestri; quella di Barcellona soltanto 3 maestri; l’istituto ispano-italiano avrebbe visto la nomina di Attilio Venturi e avrebbe mantenuto il contatto con le istituzioni spagnole attraverso il CSIC (Carlo Consiglio si sarebbe unito al professor Ippolito Galante).

Il 30 novembre 1944 il nuovo direttore della DIE, Enrico Bombieri, informò l'ambasciatore Paulucci della linea d'azione che il Ministero intendeva seguire riguardo agli insegnanti in Spagna. Mentre De Zuani doveva necessariamente cambiare destinazione, in un paese in cui il suo ricordo non fosse messo in rapporto con il fascismo, Galante avrebbe dovuto insegnare al Liceo Italiano. Il progetto di Paulucci servì come punto di riferimento definitivo per la riforma da intraprendere nelle istituzioni culturali italiane nel paese; malgrado ciò il corso del IIC nel 1944/1945 fu sostanzialmente invariato rispetto a quello dell'anno precedente. Più problematica si rivelò la situazione delle scuole a Madrid, dove si creò una commissione per affrontare il problema del debito e ottenere fondi sufficienti per proseguire le attività della scuola nel corso dell'anno 1944/1945 ${ }^{31 .}$

\section{Un bilancio della vicenda}

L'analisi della politica culturale fascista in Spagna, ha evidenziato l'interesse del fascismo per porre la cultura italiana allo stesso livello di quelle delle grandi potenze, in particolare quella francese e quella tedesca. Un progetto che aveva bisogno di grandi sforzi e che fu condizionato dallo sviluppo politico interno alla Spagna.

Come abbiamo visto questa politica non fu monolitica durante il ventennio, poiché furono cambiati gli obiettivi e le azioni nell'ambito del progetto generale. Così, durante i primi anni di governo, fino a quando non si produsse un consolidamento interno, si mantenne una linea di continuità rispetto alle politiche portate avanti dallo Stato

\footnotetext{
${ }^{30}$ Ibidem.

${ }^{31}$ ID., Situazione delle istituzioni culturali in Spagna all'8 novembre 1944. Su questo argomento cfr. LLERA ESTEBAN, Luis de, Relaciones culturales italo-hispánicas: La embajada de T. Gallarati Scotti en Madrid (1945-1946), Milán, Cisalpino-Goliardica, 1985.
} 
liberale: l'azione veniva condizionata dalle richieste e rivendicazioni effettuate dalla comunità italiana in Spagna. Tuttavia non si può dimenticare che, prima che fossero approvate le leggi fascistissime del 1925 e 1926, il regime avesse approfittato della creazione spontanea di cellule del partito all'estero per organizzare una prima macchina di propaganda coordinata da Roma attraverso la segreteria del Fasci all'Estero. Dopo aver consolidato il regime totalitario in Italia, il fascismo avviò una politica estera di maggiori dimensioni rispetto a quelle precedenti evidenziate dalla costruzione di una rete scolare più ampia, la creazione degli IIC o la creazione del IRCE - all'interno del Ministero degli Affari Esteri - e della Direzione generale per la propaganda, con competenze all'estero e sotto il controllo del Minculpop. Questa seconda fase, che può essere inquadrata nella politica culturale avviata dal fascismo all'estero, coincise con il graduale ritiro dell'Italia dalla Società delle Nazioni e con una più stretta vicinanza alla Germania nazista.

Se mettiamo a confronto la situazione della politica spagnola durante l'arco cronologico oggetto di questo studio, dobbiamo cominciare con il precisare che per il fascismo l'arrivo al potere di Primo de Rivera nel settembre 1923, aprì la possibilità di esercitare un'ascendenza culturale su un regime autoritario ampiamente interessato alle riforme effettuate in Italia. Anche se da quel momento il fattore culturale diventò un altro meccanismo al servizio dell'espansione fascista, durante questa fase la politica culturale si limitò quasi esclusivamente all'azione svolta a Barcellona, dove era presente la comunità più grande tra tutte quelle dislocate nel paese. Con l'avvento della Seconda Repubblica il fascismo si oppose al giro di boa avvenuto sulla scena politica spagnola. Mussolini non digerì i cambiamenti che impedivano di perpetuare in Spagna "l'impunità ideologica" della fase precedente: diede copertura economica ai gruppi autoctoni che cospiravano contro la Repubblica, intensificò il lavoro di propaganda, coordinato dall'Ufficio stampa del Ministero degli Affari Esteri - dal 1937 noto come Minculpop -, e gli sforzi per rafforzare la presenza culturale nel paese con la creazione di una Casa d'Italia a Madrid, l'apertura di nuovi comitati della Società Dante Alighieri nel nord della Spagna e la creazione del primo IIC a Barcellona. Finalmente, dopo la guerra civile, il fascismo si garantì una posizione culturale privilegiata nel nuovo regime. La volontà di Mussolini di aumentare il numero di organismi incaricati della diffusione della cultura italiana rappresentò la continuazione dell'aiuto militare fornito al bando nacional. In questo contesto, la cultura italiana e quella tedesca ricevettero la più alta considerazione: dovevano diventare il modello con cui riorganizzare la vita politica della nuova Spagna. La crisi dello Stato fascista e la catena di eventi che provocarono la sua caduta durante la Seconda Guerra Mondiale portarono alla 
defascistizzazione della vasta rete culturale creata nel paese e al suo progressivo smantellamento durante gli ultimi anni della guerra.

Per quanto riguarda la comunità italiana va osservato, in riferimento alla più numerosa, come l'élite di Barcellona accolse a braccia aperte la nuova ideologia perché questa rappresentava l'avvento di un potere forte, un potere che reprimeva i settori operai che si mostravano sovversivi. L'azione del Consolato e del Fascio locale fu vitale per superare le eventuali resistenze dei dirigenti delle antiche associazioni liberali - in particolare quella del presidente del comitato della Società Dante Alighieri, fondata nel 1910 - attraverso l'istituzione di un consiglio di amministrazione -che si incaricò di assumere le funzioni didattiche, assistenziali e commemorative della comunità- che fu sottoposto alla supervisione del console e che, nel 1926, diede prova di aver superato tutte le difficoltà relative al suo funzionamento. Nel resto del paese, con colonie più piccole, il raggruppamento dei connazionali e la creazione di fasci rispose più alle iniziative particolari che alla volontà del PNF. Se durante la Seconda Repubblica furono costretti ad abbandonare gli spazi pubblici dovendo rifugiarsi negli edifici consolari per l'arrivo di centinaia di antifascisti italiani dalla Francia - questi ultimi sfidavano il loro status di sinceri rappresentanti dell'italianità - con la sollevazione militare diventò più visibile la loro presenza: nelle zone controllate dai ribelli giustificarono significativamente la propaganda relativa all'intervento italiano. Così, mentre da un lato questi gruppi erano contestati all'interno del regime fascista, in Spagna venivano gratificati dal bando nacional, stabilendo stretti legami con la Falange.

Per quanto riguarda le istituzioni culturali, occorre rilevare come le scuole italiane nel paese iberico aderirono alla legislazione fascista a Barcellona e Madrid. Nei due casi si occuparono dell'insegnamento a livello elementare e medio. Gli sforzi per istituire le scuole di Barcellona risalgono al 1877 - lì funzionava una scuola elementare dall'anno scolastico 1881/1882 - mentre a Madrid cominciarono a funzionare soltanto nel 1940. Il tempo libero degli studenti venne organizzato all'estero mediante diverse attività esterne al curriculum -escursioni, recital o eventi sportivi - a seconda dell'età e del sesso dei giovani. In termini quantitativi, i risultati ottenuti dalle scuole risultarono peggiori del previsto, in particolar modo perché non risultarono in grado di attirare l'interesse della popolazione spagnola che non percepiva il prestigio di questi centri allo stesso livello di quello difeso dai rappresentanti italiani nel paese.

Per la seconda di queste istituzioni, l'IIC, tralasciando il primo tentativo effettuato a Barcellona nel 1934, la fine della Guerra Civile permise di stabilire le condizioni necessarie per l'inserimento a Madrid di una sede centrale che diventò lo strumento unificante di tutta la politica culturale italiana nel paese. La messa in opera dell'IIC fu 
affidata a Salvatore Battaglia, che organizzò i corsi di lingua, letteratura e cultura italiana nelle università -come fedele alle direttive della DIE e del Ministerio de Educación Nacional - e nelle sezioni e delegazioni del proprio istituto, che avrebbero dovute essere aperte in tutto il paese. Queste sedi furono divise in due categorie: 7 sezioni furono guidate da professori nelle città universitarie di Barcellona, Salamanca, San Sebastian, Siviglia, Valenza, Valladolid e Saragozza; in 12 delegazioni la direzione fu conferita a maestri: nelle città di Bilbao, Burgos, La Coruña, Gijón, Granada, Malaga, Oviedo, Pamplona, Santander, Santiago di Compostela, Vigo e Tetuán. La spiegazione grandezza di questa struttura è giustificata dal fatto che la Spagna "fu beneficiata" dalla riduzione del numero di rapporti amichevoli del fascismo. La Spagna diveniva uno scopo "obbligatorio" della sua politica culturale. La figura del direttore dell'IIC acquisì grande rilievo nella progettazione dell'azione italiana: a lui sarebbe spettato il compito di controllare tutte l'altre sedi e pianificare le attività che in esse si sarebbe dovuta sviluppare nei successivi anni accademici. Dopo il numero elevato di matricole registrate nel corso 1939-1940, la partecipazione si ridusse gradualmente, ma in modo significativo nelle delegazioni, rendendo molto difficile giustificare l'apertura di 20 centri per soli 2360 studenti nel corso dell'anno 1942/1943: peraltro quasi il 50\% di questi era iscritto alle sedi di Madrid e Barcellona.

Un altro obiettivo perseguito con tenacia dal fascismo, l'inserimento dell'insegnamento della lingua italiana nel sistema educativo spagnolo, era legato alla politica educativa dei governi spagnoli. Nel settore dell'istruzione secondaria l'adozione del Piano Callejo nel 1926 aumentò il numero di ore dedicate allo studio delle lingue moderne, tra cui si trovavano il tedesco, l'inglese e l'italiano. Nel 1931, tuttavia, quando fu abrogato il piano, l'italiano scomparve dal curriculum. Nella Spagna nazionale il programma di studi approvato nel 1938 introduceva nuovamente lo studio obbligatorio di una lingua romanza nella scuola media. Il vantaggio iniziale di questa misura fu vanificato dalla ridotta possibilità che avevano gli studenti di scegliere tra francese o italiano: da una parte a causa della mancanza di docenti per impartire l'insegnamento; per ovviare all'inconveniente il governo italiano si offrì di formare gli insegnanti con l'avvio di Cursillos straordinari. A livello universitario le riforme repubblicane cancellarono la presenza dellitaliano in questi centri dal momento che erano stati soppressi gli studi di Letteratura Moderna creati durante la dittatura di Primo de Rivera. Al loro posto si creò un curriculum di Filologia Moderna con due itinerari: uno per la filologia spagnola e un altro per diverse lingue straniere - francese, tedesco, inglese, portoghese e italiano- che aveva in comune il sistema di valutazione. Già nella guerra civile, il governo italiano chiese al Ministerio de Educación Nacional 
l'introduzione di diverse misure improntate alla reciprocità: l'istituzione regolare di cattedre, l'arrivo di professori per impartire corsi e seminari, la creazione di lettorati presso le università e l'erogazione di borse di studio per perfezionare la lingua. Le successive modifiche apportate dalla legislazione franchista dalla metà degli anni Quaranta limitarono l'insegnamento della lingua italiana alle sezioni di Filologia Romanza delle facoltà di Lettere e Filosofia; per l'anno accademico 1945/1946 la presenza dell'insegnamento dell'italiano fu limitato ai lettori delle tre università che avevano mantenuto il corso: Madrid, Barcellona e Salamanca.

Gli effetti della politica culturale nella sua missione di proselitismo produssero risultati molto limitati nella vita pubblica prima della ribellione militare, come si evince dalla poca attività svolta dal CAUR spagnolo; tuttavia, non può essere ignorato il fatto che i ribelli avessero rivolto il loro sguardo verso l'Italia e che vari elementi dell'ideologia fascista fossero stati recepiti dai gruppi socio-politici che sostennero il colpo di Stato. In ogni caso, come in altri aspetti, il fascismo dovette attendere fino al 1939 per diventare il modello politico da imitare nella riorganizzazione del paese. La presenza di ispanisti di prestigio nelle istituzioni culturali italiane nel paese o la visita nella penisola Iberica di grandi autorità come Arturo Farinelli, Luigi Fantappiè, Umberto Nobile, Luigi Federzoni o Amedeo Fani facilitarono l'approccio con il mondo accademico spagnolo, particolarmente attento nel settore del Diritto. A questo approccio contribuirono anche il sistema di borse di studio per gli studenti, i rapporti con il SEU e la visita di insegnanti spagnoli a centri culturali italiani. Nella stessa ottica, le attività culturali e ricreative sviluppate come complemento della vita accademica quotidiana delle scuole e dell'IIC - eventi come esposizioni, mostre, conferenze, proiezioni di film, concerti, visite e commemorazioni - servirono per cementare il senso comunitario della comunità italiana e per influenzare le relazioni bilaterali carpendo le simpatie della società spagnola.

L'obiettivo principale della politica culturale durante la dittatura di Primo de Rivera fu il controllo della sociabilità della comunità emigrata, mentre nella Seconda Repubblica, il contesto politico, lungi da spaventare Mussolini, fu l'incentivo per disegnare un piano con cui aumentare gradualmente il numero di istituzioni culturali che sviluppavano attività di propaganda. Dopo la vittoria di Franco e la fine della Guerra Civile questa politica fu modificata, in contrasto con la teoria esposta da Coverdale sulla mancanza di obiettivi ideologici di Mussolini in Spagna ${ }^{32}$. Di

\footnotetext{
32 «Salvo los incidentes en torno a la misión de Farinacci en el primer trimestre de 1937, Roma no hizo ninguna tentativa seria de inducir a los nacionales españoles a seguir modelos fascistas, y tampoco trató de reforzar y apoyar a los grupos profascistas dentro de la coalición nacional.
} 
conseguenza, il possibile "carattere difensivo" dell'intervento militare in Spagna per impedire un'alleanza tra i due fronti popolari fu modificato in corsa durante la guerra per dare il via ad un'offensiva culturale. L'IIC, come organismo che doveva rappresentare culturalmente l'Italia, giocò il ruolo di collegamento tra le autorità centrali e quelle periferiche, tra i lettori e i docenti di lingua italiana, tra le università e le istituzioni culturali spagnole; gestì borse di studio e scambi universitari. Questi incarichi, senza dubbio, fecero dell'IIC l'organismo culturale con maggiori finalità propagandistiche tra quelli creati in Spagna dai paesi stranieri. Con l'armistizio del settembre 1943 fu riformulata la politica culturale sino ad allora sviluppata: un preludio del nuovo corso che si sarebbe aperto dopo la Seconda Guerra Mondiale nelle relazioni bilaterali. 


\section{* L'autore}

Rubén Domínguez Méndez è Dottore Europeo in Storia Contemporanea sotto la supervisione della Prof.ssa Elena Maza Zorrilla (Università di Valladolid) e del Prof. Maurizio Ridolfi (Università della Tuscia). È autore di numerosi articoli sulla politica culturale italiana all'estero, specialmente durante l'epoca fascista. Recentemente ha pubblicato il saggio: Mussolini y la exportación de la cultura italiana a España, Madrid, Arco Libros, 2012.

URL: < http://studistorici.com/progett/autori/\# DomínguezMéndez >

\section{Per citare questo articolo:}

DOMÍNGUEZ MÉNDEZ, Rubén, «Note sulla politica culturale del fascismo in Spagna (1922-1945)», Diacronie. Studi di Storia Contemporanea: Sulle tracce delle idee, 29/12/2012,

URL:< http://www.studistorici.com/2012/12/29/dominguez-mendez_numero_12/ >

Diacronie Studi di Storia Contemporanea $\beta$ www.diacronie.it

Risorsa digitale indipendente a carattere storiografico. Uscita trimestrale. redazione.diacronie@hotmail.it

Comitato di redazione: Marco Abram - Jacopo Bassi - Luca Bufarale - Gianluca Canè - Alessandro Cattunar - Alice De Rensis - Barbara Galimberti - Deborah Paci - Fausto Pietrancosta - Matteo Tomasoni - Luca Zuccolo

Diritti: gli articoli di Diacronie. Studi di Storia Contemporanea sono pubblicati sotto licenza Creative Commons 2.5. Possono essere riprodotti a patto di non modificarne i contenuti e di non usarli per fini commerciali. La citazione di estratti è comunque sempre autorizzata, nei limiti previsti dalla legge. 